\title{
Investigation of the Appropriate Partial Level Density Formula for Pre-Equilibrium Nuclear Exciton Model
}

\author{
Shafik Shakir Shafik ${ }^{1 *}$, Ali Dawoud Salloum ${ }^{2}$ \\ ${ }^{1}$ Department of Physics, College of Science, University of Baghdad, Baghdad, Iraq \\ ${ }^{2}$ Department of Physics, College of Science for Women, University of Baghdad, Baghdad, Iraq \\ Email: *shafeq_sh@yahoo.com
}

Received June 10, 2013; revised July 15, 2013; accepted August 20, 2013

Copyright (C) 2013 Shafik Shakir Shafik, Ali Dawoud Salloum. This is an open access article distributed under the Creative Commons Attribution License, which permits unrestricted use, distribution, and reproduction in any medium, provided the original work is properly cited.

\begin{abstract}
Ericson formula represents the first formula, which was suggested to describe the partial level density (PLD) formula in pre-equilibrium region of the nuclear reactions. Then a number of corrections were added to this formula in order to make it more suitable to physical meaning. In this paper, there are two aims to be done: the first aim is to study the correspondence between one and two-components formulae in Ericson, Pauli, and pairing corrections; the second aim is to compare and study the results of Comprehensive formula, which contents with all corrections, with Ericson, Pauli, and pairing formulae. The Comprehensive formula was suggested to simulate the reality. To achieve these aims the ${ }^{56} \mathrm{Fe}$ and ${ }^{90} \mathrm{Zr}$ nuclei were chosen and the results showed that the difference between one and two-components formulae was too small which can be neglected. Furthermore, the results strongly recommended that for cross section calculations of the nuclear reaction, one must use Comprehensive formula rather than Pauli formula.
\end{abstract}

Keywords: Partial Level Density; Pre-Equilibrium Model; Nuclear Reaction; Exciton Model; Equidistant Spacing Model $;{ }^{56} \mathrm{Fe} ;{ }^{90} \mathrm{Zr}$

\section{Introduction}

Nuclear reactions are divided according to the energy of the incident particle to low, medium, and high-energy reactions. Pre-Equilibrium (PE) region is a stage in nuclear reaction, which represents the case when the excitation energy is not distributed equally between the nucleons of the excited nucleus. Many models describe this region such as: the Intra-Nuclear-Cascade (INC) model [1], the Harp-Miller-Berne (HMB) model [2,3], the Hybrid model [4], the Geometry Dependent Hybrid model [5] and the Exciton model [6]. J. J. Griffin suggested the Exciton model at 1966 [6]. It was preferred model by many researchers $[7,8]$ because it is simplest in the description and treatment of the PE nuclear emission. The idea of this model supposes that when the bombarding particle hit the target nucleus, it begins to share its energy with the first particle collides with it, then by successive nucleon-nucleon interactions in a series of stages and before attend to the complete interactions (equilibrium), the nuclear emission occurs. Each interaction

\footnotetext{
"Corresponding author.
}

produces a particle-hole $(p-h)$ pair, and the sum of such particle and hole called an Exciton. The first few states are $2 p 1 h, 3 p 2 h$ and so on. The numbers of excitons are $n(n=p+h)$ and the stages are labeled by $s$ so that $n=$ $2 s+1$, therefore, again, through exciton production, the nuclear emission may occur [9].

\section{The Partial Level Density (PLD)}

The PLD represents important quantity in nuclear physics. It is used in calculation of cross section, double differential cross section and transition rates $[9,10]$. PLD can be measured experimentally up to $15 \mathrm{MeV}$; above this value of energy, the levels converge. Therefore, the spacing between them overlapped, and it is difficult to calculate them. Hence, level density may be calculated by theoretical methods [8-10]. In this paper, the theoretical description of PLD by the exciton model was depended and developed through many stages. The Fermi gas model (FGM) was used as description model in the nuclear reaction exciton model. FGM conceder equal spaces between the levels and this called Equidistant Spacing Model (ESM) $[9,10]$. 
Ericson's formula is the first formula which describes the PLD and it is represents the crud formula

$$
\omega_{1}(n, E)=\frac{g^{n} E^{n-1}}{p ! h !(n-1) !}
$$

where, sign 1 means one component, i.e. the particles are not separated to protons and neutrons, $g$ is the singleparticle state density, $n$ is the exciton number, $p$ is the particles' number, $h$ is the holes' number and $E$ is the excitation energy.

The two-component formula is

$$
\omega_{2}(n, E)=\frac{g_{\pi}^{n_{\pi}} g_{v}^{n_{v}}(E)^{n-1}}{p_{\pi} ! h_{\pi} ! p_{v} ! h_{v} !(n-1) !}
$$

The two-component formula, with sign 2, distinguish between proton particles $\left(p_{\pi}\right)$ and neutron particles $\left(p_{v}\right)$ and proton holes $\left(h_{\pi}\right)$ and neutron holes $\left(h_{v}\right)$. $g_{\pi}$ and $g_{v}$ are the single particle state densities of proton and neutron, respectively, while $n_{\pi}$ and $n_{v}$ are the proton and neutron excitons numbers, $n=n_{\pi}+n_{v}$. However, $\pi$ and $\boldsymbol{v}$ stand for protons and neutrons respecttively.

In order to make the results of the PLD more reality, the Ericson's PLD formula was developed by adding physical corrections to it. Those corrections are

\subsection{Pauli's Correction}

This correction comes from Pauli's principle that forbids any two particles from having the same quantum state. So, the excitation energy deceases by the factor called Pauli Blocking Factor $A_{(p, h)}$ which given by [11]

$$
A_{(p, h)}=\frac{p(p+1)+h(h-3)}{4 g}
$$

Then the one-component PLD formula becomes

$$
\omega_{1}(n, E)=\frac{g^{n}\left(E-A_{(p, h)}\right)^{n-1}}{p ! h !(n-1) !} \Theta\left(E-A_{(p, h)}\right)
$$

$\Theta\left(E-A_{(p, h)}\right)$ is the Heaviside step function defined as

$$
\Theta\left(E-A_{(p, h)}\right)= \begin{cases}0 & \text { if } E-A_{(p, h)} \leq 0 \\ 1 & \text { if } E-A_{(p, h)}>0\end{cases}
$$

In the case of two-component, the Pauli Blocking factor is

$$
\begin{aligned}
& A_{p_{\pi}, h_{\pi}, p_{v}, h_{v}} \\
& =\left(\frac{p_{\pi}\left(p_{\pi}+1\right)+h_{\pi}\left(h_{\pi}-3\right)}{4 g_{\pi}}-\frac{p_{v}\left(p_{v}+1\right)+h_{v}\left(h_{v}-3\right)}{4 g_{v}}\right)
\end{aligned}
$$

Then the PLD for two-component can be given as

$$
\begin{aligned}
& \omega_{2}(n, E) \\
& =\frac{g_{\pi}^{n_{\pi}} g_{v}^{n_{v}}\left(E-A_{p_{\pi}, h_{\pi}, p_{v}, h_{v}}\right)^{n-1}}{p_{\pi} ! h_{\pi} ! p_{v} ! h_{v} !(n-1) !} \Theta\left(E-A_{p_{\pi}, h_{\pi}, p_{v}, h_{v}}\right)
\end{aligned}
$$

\subsection{Pairing Correction}

This correction comes from pairing property between couples of particles. Therefore, this pairing required energy, which is taken from the excitation energy and hence the excitation energy will decrease [12-14]. Thus, the PLD formula in one component with pairing correction can be given by (all details of this correction given by these references) $[9,11]$

$$
\omega_{1}(n, E)=\frac{g^{n}\left(E-P(\Delta)-B_{p, h}\right)^{n-1}}{p ! h !(n-1) !} \Theta\left(E-P(\Delta)-B_{p, h}\right)
$$

where $P(\Delta)$ is the pairing correction, and it is given by

$$
P(\Delta)=\frac{\left(\Delta_{o}^{2}-\Delta^{2}\right)}{4} g
$$

$\Delta_{o}$ and $\Delta$ are the energy gaps of the ground and excited states, respectively.

$B_{p, h}$ is the modified Pauli Blocking factor which is given by

$$
B_{p, h}=A_{p, h} \sqrt{1+\left(\frac{2 g \Delta}{n}\right)^{2}}
$$

$A_{p, h}$ is unmodified Pauli Blocking factor given by Equation (3). $\Delta$ is given by

$$
\frac{\Delta}{\Delta_{o}}= \begin{cases}0.996-1.76\left(\frac{n}{n_{c}}\right)^{1.6}\left(\frac{E}{C_{e}}\right)^{-0.68} & \text { if } E \geq E_{\text {phase }} \\ 0 & \text { if } E<E_{\text {phase }}\end{cases}
$$

where $n_{c}$ is the most probable exciton number that leads to emission

$$
n_{c}=0.792 g \Delta_{o}
$$

$C_{e}$ is the condensation energy given by

$$
C_{e}=g \frac{\Delta_{o}}{4}
$$

$E_{\text {phase }}$ is the pairing energy of phase transition defined as following

$$
E_{\text {phase }}= \begin{cases}C_{e}\left[0.716+2.44\left(\frac{n}{n_{c}}\right)^{2.17}\right] & \text { if } \frac{n}{n_{c}} \geq 0.446 \\ 0 & \text { otherwise }\end{cases}
$$


The minimum value of energy for applying these equations is the effective value of energy, which means threshold value of $E_{\text {phase }}$ and it is given by equations

$$
U_{t h}= \begin{cases}C_{e}\left[3.23 \frac{n}{n_{c}}-1.57\left(\frac{n}{n_{c}}\right)^{2}\right] & \text { if } \frac{n}{n_{c}} \geq 0.446 \\ C_{e}\left[1+0.627\left(\frac{n}{n_{c}}\right)^{2}\right] & \text { if } \frac{n}{n_{c}}>0.446\end{cases}
$$

The value of $\Delta_{o}$ can be obtained from curve fitting for almost known nuclei, by a relation known as GilbertCameron formula

$$
\Delta_{o}=\Delta_{o \pi}+\Delta_{o v}
$$

where $\Delta_{о \pi}$ and $\Delta_{o v}$ are the energy gaps for ground states of proton's and neutron's particles, respectively.

$$
\begin{aligned}
& \Delta_{o \pi}=1.654-9.58 \mathrm{Z} \times 10^{-3} \\
& \Delta_{o v}=1.374-5.16 \mathrm{~N} \times 10^{-3}
\end{aligned}
$$

$N$ and $Z$ are the neutrons and protons numbers respecttively.

The value of $\Delta_{o}$ is depending on the nuclear temperature, where it increases with temperature and vanishes at a critical temperature which gives for ESM by $\frac{2 \Delta_{o}}{3.5}$ [11]. Above this temperature, the pairing correction disappeared and the system reverted to uncorrelated condition. The two-component formula is given by

$$
\begin{aligned}
& \omega_{2}(n, E)=\frac{g_{\pi} g_{v}\left(E-P_{2}(\Delta)-B_{p_{\pi} h_{\pi} p_{v} h_{v}}\right)^{n-1}}{p_{\pi} ! h_{\pi} ! p_{v} ! h_{v} !(n-1) !} \\
& \Theta\left(E-P_{2}(\Delta)-B_{p_{\pi} h_{\pi} p_{v} h_{v}}\right)
\end{aligned}
$$
by

$P_{2}(\Delta)$ is the two-components pairing energy given

$$
\begin{aligned}
& P_{2}(\Delta)=P_{1}\left(\Delta_{\pi}\right)+P_{1}\left(\Delta_{v}\right) \\
& P_{1}\left(\Delta_{\pi}\right)=\frac{\left(\Delta_{o \pi}^{2}-\Delta_{\pi}^{2}\right)}{4} g_{\pi} \\
& P_{1}\left(\Delta_{\pi v}\right)=\frac{\left(\Delta_{o v}^{2}-\Delta_{v}^{2}\right)}{4} g_{v}
\end{aligned}
$$

$B_{p_{\pi} h_{\pi} p_{v} h_{v}}$ is the modified Pauli Blocking factor in the case of two component

$$
B_{p_{\pi} h_{\pi} p_{v} h_{v}}=A_{p_{\pi}, h_{\pi}, p_{v}, h_{v}} \sqrt{1+\left(\frac{2 g \Delta}{n}\right)^{2}}
$$

$A_{p_{\pi}, h_{\pi}, p_{v}, h_{v}}$ is the unmodified Pauli Blocking factor given by Equation (5).

\subsection{Active and Passive Holes Correction}

The exciton model assumed that the particle's number must be equal to the holes number, but in fact, the particles number is always bigger than the holes number by one. This is because the projectile (incident particle) is added to the particles number. In order to satisfy the equality between particles number and holes number, passive holes have been supported. They represent those holes are not affected by the nuclear potential; therefore, accumulated near Fermi's level. The correction that comes from passive holes was given by $[9,15]$

$$
\begin{gathered}
A_{k p, h}=\frac{q^{2}}{g}-\frac{p(p+1)+h(h+1)}{4 g} \\
q=\max (p, h)
\end{gathered}
$$

Then, the PLD can take the form

$$
\omega_{1}(n, E)=\frac{g^{n}\left(E-A_{k, p h}\right)^{n-1}}{p ! h !(n-1) !} \Theta\left(E-A_{k, p h}\right)
$$

\subsection{Charge Factor Correction}

This correction takes into account the charge effect on PLD calculations. The Charge effect is represented by the effective charge factor, which had been expressed by many formulae. However, many researchers don't apply this correction $[9,16]$. Therefore, the charge factor effect was neglected in this work.

\subsection{Isospin Correction}

To add the effect of isospin in nuclear reactions, it is necessary to determine how much the isospin is conserved or mixed and what is the isospin symmetry energy?

If the isospin is included in calculation of level density, it is important to take one-to-one correspondence between states of the same isospin in isobaric nuclei [17-19].

$$
\begin{aligned}
& \omega\left(p, h, E, T, T_{z}=T\right) \\
& =\omega\left(p, h, E+E_{\text {sym }}(T, T-1), T, T-1\right) \\
& =\omega\left(p, h, E+E_{\text {sym }}(T, T-2), T, T-2\right)
\end{aligned}
$$

$E_{\text {sym }}$ is the symmetric energy which is given by empirical equation

$$
E_{\text {sym }}=\left(110 A^{-1}-133 A^{-4 / 3}\right)\left(T^{2}-T_{z}^{2}\right)
$$

The PLD formula which contains the isospin can be given by

$$
\begin{aligned}
& \omega_{1}(p, h, T, E) \\
& =\frac{g^{n}\left(E-A_{p h, T}\right)^{n-1}}{p ! h !(n-1) !} f_{T}(p, h, T) \Theta\left(E-A_{p h, T}\right) \\
& A_{p h, T}=A_{p h}+E_{s y m}\left(T, T_{z}\right)
\end{aligned}
$$


$f_{T}(p, h, T)$ is the correction factor of states with good isospin. If isospin is assumed to be completely mixed, the symmetric energy is zero and the correction factor $f_{T}$ is unity.

\subsection{Spin Correction}

Spin effect is also added to the PLD formula as a correction and it is assumed factorized [20-23]

$$
\begin{gathered}
\rho(n, E, J)=\frac{g^{n}\left(E-A_{(p, h)}\right)^{n-1}}{p ! h !(n-1) !} R_{n}(J) \Theta\left(E-A_{(p, h)}\right) \\
R_{n}(J)=\frac{2 J+1}{2 \sqrt{2 \pi} \sigma^{3}} \exp \left(-\frac{\left(J+\frac{1}{2}\right)^{2}}{2 \sigma_{n}^{2}}\right)
\end{gathered}
$$

$\sigma_{n}$ is the spin cut off parameter. It is important to mention that Equation (26) used for pre-compound nucleus and it does not use for compound nucleus [22].

$$
\sigma_{n}^{2}=0.16 n A^{2 / 3}
$$

\subsection{Surface Correction}

The initial interaction between a projectile and target nucleon is frequently localized near the nucleus surface. Since the nuclear density variant with nuclear radius, hence the nuclear potential is shallower than in the interior; therefore, the local well depth (i.e. near the surface) is less the central depth $[9,11,24]$. This must add a considerable effect on PLD calculations especially in the exciton knockout and pickup nuclear reactions.

If we labeled $\omega(n, E, V)$ as the PLD of one-component Fermi gas system with exciton number $n$, excitation energy $E$ and nuclear potential well depth $V$, then one can write the following equation for PLD with surface effect

$$
\omega_{1}(n, E, V)=\omega_{1}(n, E, \infty) \times f_{1}(n, E, V)
$$

where $\omega_{1}(n, E, \infty)$ is the PLD calculated for $V=\infty$, and it is given by the simple one-component PLD Pauli formula (Equation (4))

$$
\omega_{1}(n, E, \infty)=\frac{g^{n}\left(E-A_{p, h}\right)^{n-1}}{p ! h !(n-1) !} \Theta\left(E-A_{p, h}\right)
$$

The function $f_{1}(n, E, V)$ in Equation (29) is the correction due to surface effect in the ESM, and it is given by

$$
f_{1}(n, E, V)=\sum_{j=0}^{h}(-1)^{j} C_{j}^{h}\left[\frac{E-j V(h)}{E}\right]^{n-1}
$$

From Equation (29), the PLD formula was corrected to include the finite well depth and then was extended to include correction due to surface effect. This was done in the initial particle-nucleus interaction by replacing the nuclear potential depth $V_{0}$ by $\bar{V}_{1}$. This means instead of putting $V=V_{0}$, we put $V=\bar{V}_{1}$. The new potential depth $\bar{V}_{1}$, is defined as the "average effective well depth". The choice between $\bar{V}_{1}$ and $V_{0}$ is

$$
V= \begin{cases}V_{0}=38 \mathrm{MeV} & h>1 \\ \bar{V}_{1} & h \leq 0\end{cases}
$$

For $(n, n)$ reaction $V_{1, n n}=7 \mathrm{MeV} \quad$ [24].

\section{Comprehensive Formula}

Comprehensive formula is a formula includes all the previous corrections, except the isospin correction because the reaction was assumed to be completely mixed [17,18], then the isospin correction became unity. The aim behind suggested this formula is an attempt to get on a formula describes the PE PLD by the most accurate description (real one).

$$
\begin{gathered}
\omega_{1}(p, h, E)=\frac{g^{n}\left(E-P(\Delta)-B_{p, h}-A_{k p, h}\right)^{n-1}}{p ! h !(n-1) !} f_{1}(n, E, V) \\
R_{n}(J) \Theta\left(E-P(\Delta)-B_{p, h}-A_{k p, h}\right)
\end{gathered}
$$

\section{Results and Discussion}

It is obvious that the PLD cannot be calculated experimentally especially for excitation energy more than 15 $\mathrm{MeV}$ because the states overlapped with each other's. On the other hand, and in order to increasing knowledge about the nuclear force, which represents the strongest force comparing with others, one must increase the applied excitation energy to hundred and several hundred MeVs. Therefore, PLD must be given in theoretical form. The calculated Comprehensive formula (Equation (32)) is a suitable formula for PLD estimations. This claim can be examined if one applies the Comprehensive formula of the PLD in cross section equation of any (and for any nucleus) PE nuclear models and comparing with experimental results of this cross section. However, PLD formula with Pauli's correction was used in cross section calculations by many researchers [25-27] and these researchers avoided use all corrections of the PLD to ease the programming potential. Further, in these results, Pauli's correction was stand as a reference case for PLD comparisons with all their corrections.

\subsection{Comparison between One and Two-Component}

In order to study the difference between one and twocomponent formulae of the PLD, a comparison was made 
for ${ }^{56} \mathrm{Fe}$ isotope. All used parameter are listed in Table 1. From Figures 1(a)-(c) one can see that the two-component results are less than those of one-component. This behavior is expected physically because the two-component system will have to share the energy with more entities (the entities are those due to particles and holes of the neutrons and protons). Although the neutron parti- cles and holes are considered zero, the two component results stay less than the one-component results.

From Figures 1(a)-(c) one can see that the two-component results are less than those of one-component. This behavior is expected physically because the two-component system will have to share the energy with more entities (the entities are those due to particles and holes
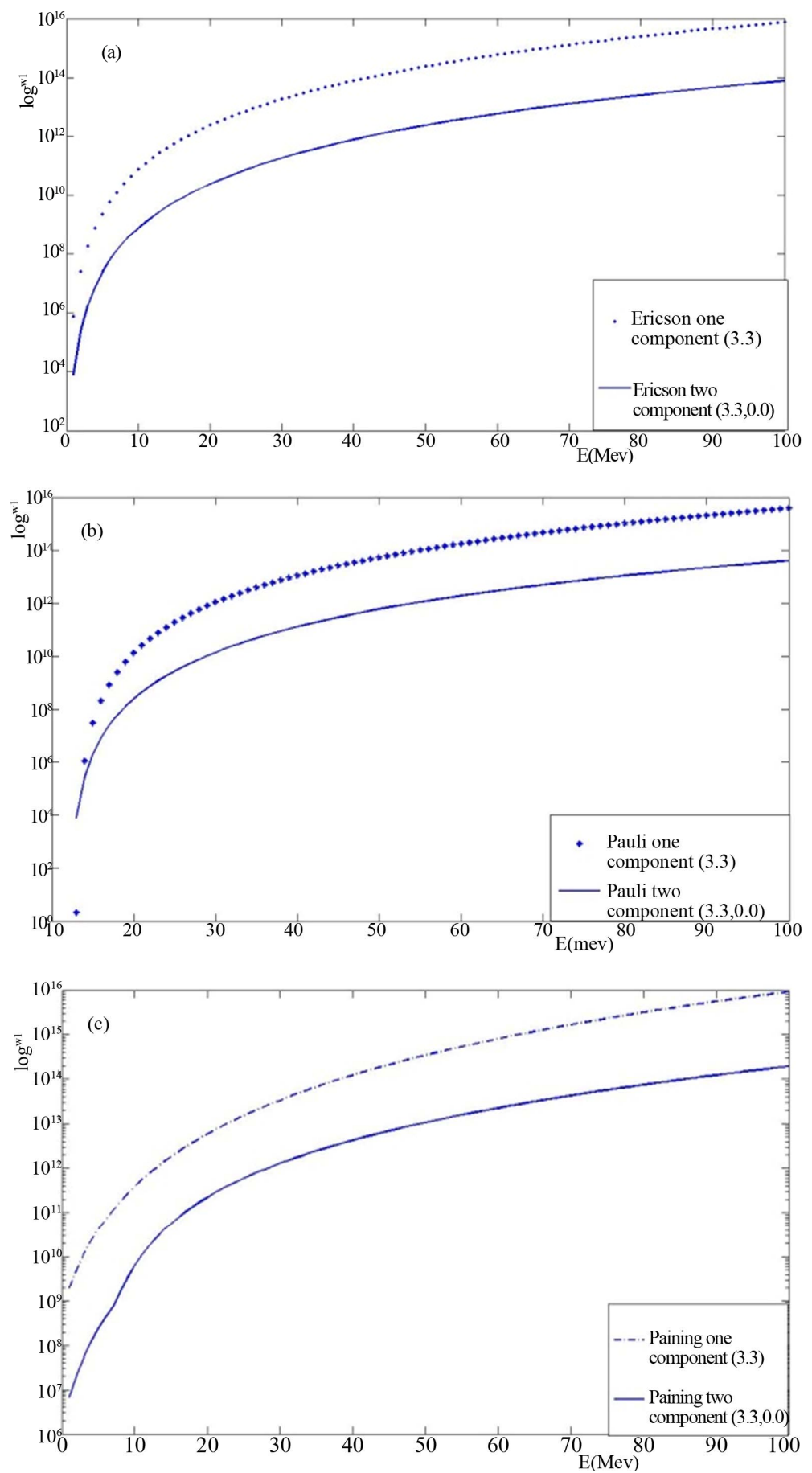

Figure 1. (a) One and two-components Ericson's formulae of PLD for ${ }^{56}$ Fe isotope; (b) One and two-components Pauli's formulae of PLD of ${ }^{56} \mathrm{Fe}$ isotope; (c) One and two-components pairing formulae of PLD for ${ }^{56} \mathrm{Fe}$ isotope. 
of the neutrons and protons). Although the neutron particles and holes are considered zero, the two component results stay less than the one-component results.

In addition, it is noted from Figures 1(a)-(c) and Table 2 that the ratio between one and two-components results is small for main forms of the PLD formulae (Ericson, Pauli, and Pairing PLD formulae), therefore, one-component formula may be used instead of twocomponent formula with acceptable results.

\subsection{Behavior of One-Component Corrections}

The PLD of one-component for Ericson formula with all corrections for ${ }^{56} \mathrm{Fe}$ isotope can be shown in Figure 2. It is noted from this figure that the arrangement of the results from bottom to top is surface correction, Pauli's correction, Ericson's correction, pairing correction, spin correction, active and passive correction and Comprehensive formula, respectively. Whereas, for ${ }^{90} \mathrm{Zr}$ isotope, shown in Figure 3, the arrangement is surface correction, Pauli correction, Ericson's correction, spin correction, active and passive holes correction, pairing correction and Comprehensive formula, respectively.

The results of these two figures give approximately same arrangements. As we mentioned above, if one used PLD with Pauli correction as reference, then it is easy to see that except surface correction, the all corrections and Comprehensive PLD formulae have values more than PLD with Pauli correction. However, one can expect that the use of PLD formula with Pauli correction alone in cross section calculations may deviate the theoretical estimations from experimental results. Therefore, these results strongly suggested that the Comprehensive PLD formulae must be used in any calculations need PLD.

Finally, the results of ${ }^{90} \mathrm{Zr}$ isotope are bigger than that of the ${ }^{56} \mathrm{Fe}$ isotope because the mass number is higher and then the single particle level density $g$ increases. On the other hand, the difference between the results decreases with increasing the excitation energy.

\section{Conclusion}

PLD represents mean arguments in nuclear reaction and stricture models. This paper submitted a good literature

Table 1. The parameter used in the present calculations.

\begin{tabular}{cc}
\hline Target nucleus under investigation & ${ }^{56} \mathrm{Fe}$ \\
\hline Mass number & 56 \\
Atomic number & 26 \\
Neutrons number & 30 \\
Exciton number & 6 \\
Exciton configuration & $(3,3)$ for one-component \\
Max. excitation energy & $100 \mathrm{MeV}$ \\
Single particle density & $\mathrm{A} / 13$ \\
\hline
\end{tabular}

Table 2. The ratio between one and two-component formulae for Ericson, Pauli and pairing PLD.

\begin{tabular}{cc}
\hline The correction & $\begin{array}{r}\text { The ratio between two and one } \\
\text { components } \omega_{2} / \omega_{1}\end{array}$ \\
Ericson & 0.007 \\
Pauli & 0.015 \\
Pairing & 0.02 \\
\hline
\end{tabular}

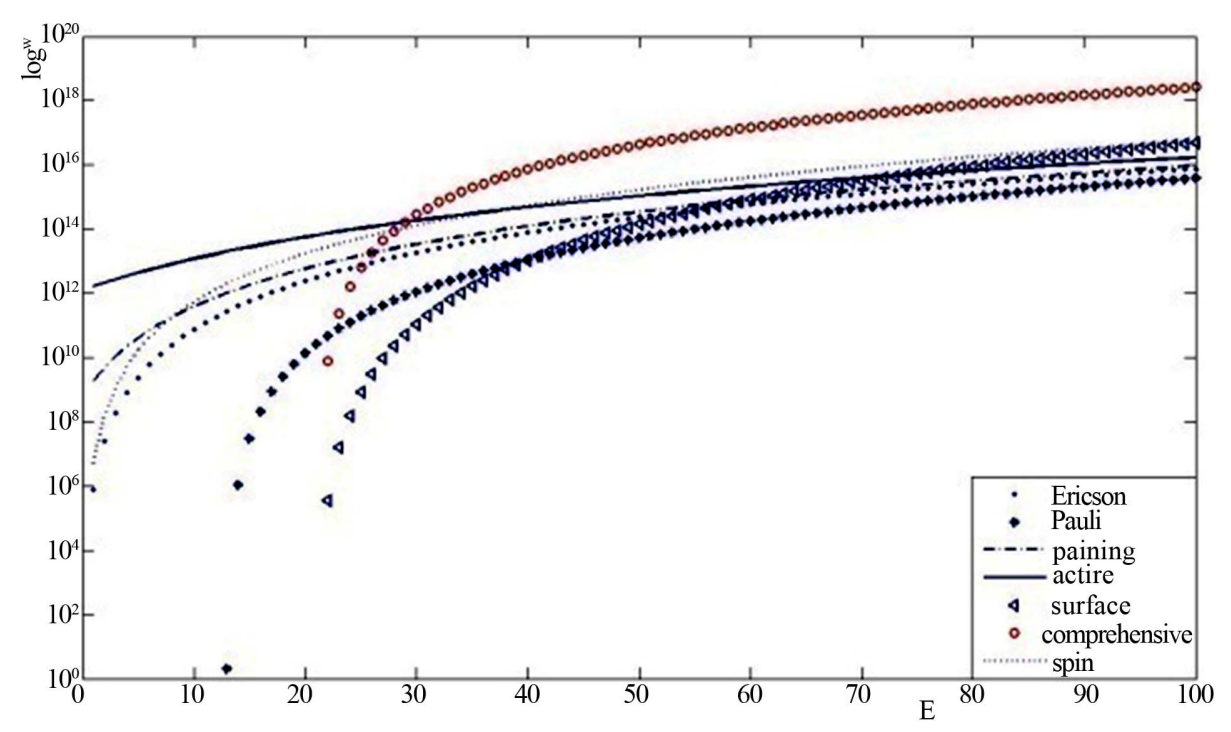

Figure 2. PLD of one-component with all corrections for ${ }^{56} \mathrm{Fe}$ isotope. 


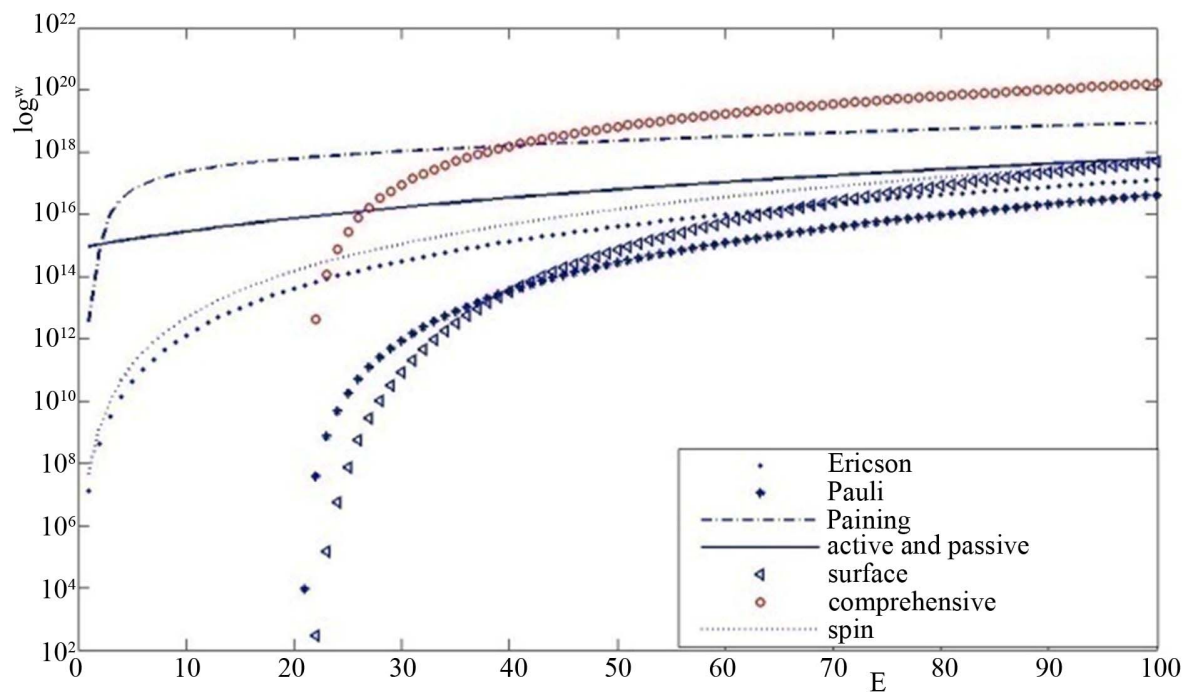

Figure 3. PLD of one-component with all corrections for ${ }^{90} \mathrm{Ze}$ isotope.

survey for the PLD formulae with its corrections and suggested a formula containing all corrections which is the "Comprehensive Formula". However, the results of this work strongly recommended that if one needs to use PLD, then the Comprehensive formula must be applied rather than Pauli formula. In addition, the results showed that the one-component formulae of PLD can be used instead of two components with acceptance results and the PLD values increase with the mass number of the target nucleus and with excitation energy of the incident particle.

\section{REFERENCES}

[1] K. K. Gudima, S. G. Mashnik and V. D. Toneev, "Cascade-Exciton Model of Nuclear Reactions," Nuclear Physics A, Vol. 401, No. 2, 1983, pp. 329-361. doi:10.1016/0375-9474(83)90532-8

[2] G. D. Harp, J. M. Miller and B. J. Berne, "Attainment of Statistical Equilibrium in Excited Nuclei," Physical Review, Vol. 165, No. 4, pp. 1166-1169. doi:10.1103/PhysRev.165.1166

[3] G. D. Harp and J. M. Miller, "Precompound Decay from Time-Dependent Point of View," Physical Review C, Vol. 3, No. 5, 1971, pp. 1847-1855. doi:10.1103/PhysRevC.3.1847

[4] M. Blann, "Importance of Nuclear Density Distribution on Pre-Equilibrium Decay," Physical Review Letters, Vol. 28, No. 12, 1972, pp. 757-759. doi:10.1103/PhysRevLett.28.757

[5] M. K. Bhardwaj and I. A. Rizivi, "Alpha-Induced Reactions in Iridium," Physical Review C, Vol. 45, No. 5, 1992, pp. 2338-2348. doi:10.1103/PhysRevC.45.2338

[6] J. J. Griffin, "Statistical Model of Intermediate Structure," Physical Review Letters, Vol. 17, No. 9, 1966, pp. 478481. doi:10.1103/PhysRevLett.17.478
[7] C. K. Cline and M. Blann, "The Pre-Equilibrium Statistical Model: Description of the Nuclear Equilibration Process and Parameterization of the Model," Nuclear Physics A, Vol. 172, No. 2, 1971, pp. 225-259. doi:10.1016/0375-9474(71)90713-5

[8] C. Kalbach, "Two-Component Exciton Model: Basic Formalism Away From Shell Closures," Physical Review C, Vol. 33, No. 3, 1986, pp. 818-833. doi:10.1103/PhysRevC.33.818

[9] E. Běták and P. E. Hodgson, "Particle-Hole State Density in Pre-equilibrium Nuclear Reactions," University of Oxford, CERN Libraries, Geneva, 1998.

[10] F. C. Williams Jr., "Particle-Hole State Density in the Uniform Spacing Model," Nuclear Physics A, Vol. 166 No. 2, 1971, pp. 231-240. doi:10.1016/0375-9474(71)90426-X

[11] M. Avrigeanu and V. Avrigeanu, "Partial Level Density for Nuclear Data Calculations," Computer Physics Communications, Vol. 112, No. 2-3, 1998, pp. 191-226.

[12] W. E. Meyrhof, "Elements of Nuclear Physics," Mc Grew Hill, New York, 1967, pp. 36,58.

[13] A. de-Shalit and I. Talmi, "Nuclear Shell Theory," Academic Press, Waltham, 1963, p. 27.

[14] A. S. Davydov, "Quantum Mechanics," Pergamon Press, Oxford, 1969, p. 371.

[15] C. Kalbach, "Missing Final States and the Spectral Endpoint in Exciton Model Calculations," Physical Review C, Vol. 73, No. 2, 2006, Article ID: 024614. doi:10.1103/PhysRevC.73.024614

[16] G. M. Braga-Marcazzan, E. Gadioli-Erba, L. M. Millazo-Colli and P. G. Sona, "Analysis of the Total (n,p) Cross Sections around $14 \mathrm{MeV}$ with the Pre-Equilibrium Exciton Model," Physical Review C, Vol. 6, No. 4, 1972, pp. 1398-1407. doi:10.1103/PhysRevC.6.1398

[17] C. Kalbach, "Isospin Dependence of Two-Component Particle-Hole State Density," Physical Review C, Vol. 30, No. 4, 1984, pp. 1310-1319. 
doi:10.1103/PhysRevC.30.1310

[18] C. Kalbach, "Particle-Hole State density with Good Isospin," Physical Review C, Vol. 47, No. 2, 1993, pp. 587601. doi:10.1103/PhysRevC.47.587

[19] C. Kalbach, "Isospin Conversion in Preequilibrium Reactions," Physical Review C, Vol. 72, No. 2, 2005, Article ID: 024607. doi:10.1103/PhysRevC.72.024607

[20] C. Kalbach and F. M. Mann, "Phenomenology of Continuum Angular Distributions. I. Systematics and Parametrization," Physical Review C, Vol. 23, No. 1, 1981, pp. 112-123. doi:10.1103/PhysRevC.23.112

[21] C. Kalbach, "Phenomenology of Continuum Angular Distributions. II. Griffin Preequilibrium Model," Physical Review C, Vol. 23, No. 1, pp. 124-135. doi:10.1103/PhysRevC.23.124

[22] M. B. Chadwick and P. Obložinský, "Particle-Hole State Densities with Linear Momentum and Angular Distributions in Preequilibrium Reaction," Physical Review C, Vol. 46, No. 5, 1992, pp. 2028-2041. doi:10.1103/PhysRevC.46.2028

[23] S. S. Shafik, "Angular Momentum Distribution for State
Density with Non-ESM Dependence," Journal of Fisica Nucleare, 2009.

[24] Ye. A. Bogila, V. M. Kolomietz, A. I. Sanzhur and S. Shlomo, "Preequilibrium Decay in the Exciton Model for Nuclear Potential with a Finite Depth," Physical Review C, Vol. 53, No. 2, 1996, pp. 855-859. doi:10.1103/PhysRevC.53.855

[25] M. Avrigeanu, A. Harangozo and V. Avrigeanu, "Average Strength of the Effective Interaction in Multistep Direct Reactions," Physical Review C, Vol. 56, No. 3, 1997, pp. 1633-1636. doi:10.1103/PhysRevC.56.1633

[26] Y. K. Gupta, D. C. Biswas, Bency John, B. K. Nayak, A. Saxena and R. K. Choudhury, "Nuclear Level-Density Parameters of Nuclei in the Z 70 and A 180 Mid-Shell Regions," Physical Review C, Vol. 80, No. 5, 2009, Article ID: 054611. doi:10.1103/PhysRevC.80.054611

[27] H. Nakada and Y. Alhassid, "Publisher's Note: Isospin-Projected Nuclear Level Densities by the Shell Model Monte Carlo Method," Physical Review C, Vol. 78, No. 6, 2008, Article ID: 069907. doi:10.1103/PhysRevC.78.069907 